\title{
Physicochemical Properties of Gold Nanostructures Deposited on Glass
}

\author{
Zdenka Novotna, ${ }^{1}$ Alena Reznickova, ${ }^{1}$ Linda Viererblova, ${ }^{2}$ Jiri Kolafa, ${ }^{2}$ \\ Zdenka Kolska, ${ }^{3}$ Jan Riha, ${ }^{4}$ and Vaclav Svorcik ${ }^{1}$ \\ ${ }^{1}$ Department of Solid State Engineering, Institute of Chemical Technology Prague, 16628 Prague, Czech Republic \\ ${ }^{2}$ Department of Physical Chemistry, Institute of Chemical Technology Prague, Prague, Czech Republic \\ ${ }^{3}$ Faculty of Science, J. E. Purkyně University, Ústí nad Labem, Czech Republic \\ ${ }^{4}$ New Technologies Research Centre in West Bohemian Region, University of West Bohemia, Plzeň, Czech Republic
}

Correspondence should be addressed to Zdenka Novotna; zdenkal.novotna@vscht.cz

Received 26 November 2013; Revised 17 April 2014; Accepted 17 April 2014; Published 2 June 2014

Academic Editor: Yangchuan Xing

\begin{abstract}
Copyright ( 2014 Zdenka Novotna et al. This is an open access article distributed under the Creative Commons Attribution License, which permits unrestricted use, distribution, and reproduction in any medium, provided the original work is properly cited.

Properties of gold films sputtered onto borosilicate glass substrate were studied. UV-Vis absorption spectra were used to investigate optical parameters. XRD analysis provided information about the gold crystalline nanostructure, the texture, and lattice parameter and biaxial tension was also determined by the XRD method. The surface morphology was examined by atomic force microscopy (AFM); chemical structure of sputtered gold nanostructures was examined by X-ray photoelectron spectroscopy (ARXPS). The gold crystallites are preferentially [111] oriented on the sputtered samples. Gold deposition leads to dramatic changes in the surface morphology in comparison to pristine glass substrate. Oxygen is not incorporated into the gold layer during gold deposition. Experimental data on lattice parameter were also confirmed by theoretical investigations of nanoclusters using tight-binding potentials.
\end{abstract}

\section{Introduction}

Glass substrates have recently attracted growing interest due to the fact that they can serve as substrates for nanostructured systems with remarkable optical nonlinear properties. Glass substrates possess overall advantages as compared to many crystals or polymers. In particular, the composition of the glass can be well designed and also their fabrication is usually feasible and inexpensive [1].

Nanostructured thin metal films play nowadays quite a significant role in various material science and nanotechnology applications. In particular, a considerable attention has been drawn to the structure and properties of thin metal films deposited on nonmetal surfaces due to their attractive applications in electronic, magnetic, and optical devices [2]. Gold nanoparticles dispersed on solid surface attract great attention due to their unique optical [3], electronic [4], thermal [5], and catalytic [5, 6] properties. Gold in the form of thin films is nowadays used in a vast range of applications such as micro- and nanoelectromechanical systems (MEMS and NEMS) [7], bio- and optical sensors [8], electronic textiles [9], and bioengineering [10], as a generator of nonlinear optical properties [11], or in devices for surfaceenhanced Raman scattering [12].

Modeling of gold (and other metal) crystals and nanoclusters by molecular simulation methods soon agreed on employing semiempirical tight-binding potentials, which are able to describe both lattice parameters and surface stress [13]. Particularly, molecular dynamics investigation of approximately isometric nanoclusters [14] showed that the atoms are on average closer near surface than in the bulk. Combination of the first-principle calculations and Monte Carlo method applied to gold small nanoclusters on $\mathrm{MgO}$ (100) surface leads to results which are in agreement with previous experimental observations [15]. Similar results were obtained also for platinum [16]. 
The present study is a continuation of our previous work in which we have studied various physicochemical properties of gold nanostructures deposited on glass substrate. This work is focused on wettability, surface roughness, sheet electrical resistance, and influence of these properties on adhesion and proliferation of VSMC [17]. Theoretical simulation of $\mathrm{Au}$ nanoclusters growth during deposition on glass substrate was performed. We performed measurement of optical properties and calculation of optical band gap $\left(E_{g}^{\text {opt }}\right)$. The physical properties such as lattice parameters, intensity and orientation of the gold crystallites, and surface morphology by AFM were evaluated. Also chemical properties of deposited gold structures such as element concentration were studied by ARXPS spectra. The gold/glass structures could find applications for electronics and tissue engineering.

\section{Methods}

2.1. Materials and Modification. The gold layers were sputtered on $1.8 \times 1.8 \mathrm{~cm}^{2}$ borosilicate microscopic glass, supplied by Glassbel Ltd., CR. The surface roughness of glass, measured over the area of $1 \times 1 \mu \mathrm{m}^{2}$ and calculated as anerage value from five different measuring positions, was $R_{a}=0.34 \pm$ $0.03 \mathrm{~nm}$ [18]. The gold sputtering was accomplished on Balzers SCD 050 device from gold target (supplied by Goodfellow Ltd., UK). The deposition conditions were DC Ar plasma, gas purity of $99.995 \%$, sputtering times of 20 and 150 s, current of 10 to $40 \mathrm{~mA}$ (discharge power 3 to $15 \mathrm{~W}$ ), total Ar pressure about $5 \mathrm{~Pa}$, and the electrode distance of $50 \mathrm{~mm}$. The power density of Ar plasma in our case was $0.13 \mathrm{~W} \cdot \mathrm{cm}^{-2}$, and the average deposition rate was $0.15 \mathrm{~nm} \cdot \mathrm{s}^{-1}$. After deposition the glass substrate was cleaned with methanol (p.a.) and dried in a stream of $\mathrm{N}_{2}$. The prepared samples were stored at laboratory conditions.

2.2. Theoretical Study. We also performed a simulation study using two potentials, a classical tight-binding potential [19] and a pairwise potential designed for gold simulations [20] to confirm our experimental data.

Tight-Binding Potential. The interatomic potential is composed of a sum over all atom pairs, $\sum_{i<j}$, and a nonadditive term, $\sum_{i}$,

$$
U=\sum_{i<j} 2 A e^{p\left(r_{i j} / r_{0}-1\right)}-\sum_{i} \rho_{i}^{1 / 2},
$$

where $r_{i j}$ is the distance of atoms $i$ and $j, A, p, r_{0}$ as well as $q$ and $\xi$ below are parameters, and $\rho_{i}$ mimics the electron density:

$$
\rho_{i}=\sum_{j \neq i} \rho_{i j}=\sum_{j \neq i} \xi^{2} e^{-2 q\left(r_{i j} / r_{0}-1\right)} .
$$

The algorithm to calculate the forces and energy proceeds in two phases [13]. In the first one the density components $\rho_{i j}$ are calculated and in the second one the calculations of the additive and nonadditive forces are accomplished by summation over all atom pairs. From these pair and pair-like forces also the pressure tensor is calculated. Another way to calculate the pressure tensor is via the virtual volume and shape change method [21]. We used this method to test the algorithm.

Pair Potential. We also implemented a simpler effective pair potential designed for gold simulations [20, 22]:

$$
U=\sum_{i<j}\left(D_{21} \frac{A_{1}}{r_{i j}^{\lambda_{1}}} e^{-\alpha_{1} r_{i j}^{2}}+D_{22} \frac{A_{2}}{r_{i j}^{\lambda_{2}}} e^{-\alpha_{2} r_{i j}^{2}}\right),
$$

where $D_{i j}, A_{i}, \alpha_{i}, \lambda_{i}$ are constants. From a technical point of view this potential is simpler because the calculations can be done in one pass.

The simulations were performed in the slab geometry. A sample crystal was oriented with the (111) lattice vector perpendicular to the slab (see Figure 1). In the simulation, this vector corresponds to the $z$-axis (or vertical direction). The system is periodic in the $x$ and $y$ (horizontal) directions. To integrate the equations of motion, we used the Verlet method with a timestep of $5 \mathrm{fs}$. The total simulation time for one sample was $7.5 \mathrm{~ns}$. In order to avoid problems with slow relaxation of slower vibrational modes, we used the Andersen thermostat [21] with a relaxation time of 1 ps. Atoms are randomly visited by a demon changing their velocity to a new one drawn from the Maxwell-Boltzmann distribution. Components of the pressure tensor in the $x$ and $y$ directions were independently kept constant by a Berendsen-type barostat [21] with a time constant of 3 ps (based on the bulk modulus of gold $180 \mathrm{GPa}$ ).

The slab geometry does not contain any substrate-gold nanolayer interaction, which could possibly affect the layer thickness. In principle, one should employ an atomistic model of a substrate, which can be rough and in turn study the whole range of relaxation processes occurring during the deposition. However, such a study lies outside of the scope of this work. The contact angles of gold alloys on silica and other substrates are around $140^{\circ}$ [23], suggesting relatively weak substrate-gold forces. As a simple model of this interaction, we considered an ideally smooth wall, composed of uniformly dispersed atoms in the $z<0$ halfspace. To build the model, we started from the gold-water oxygen interaction, which was approximated by the LennardJones 12-6 potential in [24]. The relatively high value of the energy parameter $\varepsilon=0.59 \mathrm{kcal} / \mathrm{mol}$ (with respect to the oxygen-oxygen term of $0.15-0.2 \mathrm{kcal} / \mathrm{mol}$ in common water models) takes effectively into account the interaction of the water dipole moment with metal by the mechanism of charge inversion. The silica unit of O-Si-O has several times higher dipole moment than a water molecule; we thus estimate the parameter $\varepsilon$ of the substrate (borosilicate glass) as three times that of water. In addition, we increase the atom size to $3.85 \AA$ to take into account the bigger silicon atom (3.6 $\AA$ [24] scaled by the cube root of the $\mathrm{Si}: \mathrm{O}$ diameter ratio taken from the UFF [25]). The final gold-substrate interaction energy is integrated over $z<0$ and glass density to yield the potential of the form $A / z^{9}-C / z^{3}$, where $A=2021 \mathrm{kcal} / \mathrm{mol} \AA^{9}$ and $C=4.65 \mathrm{kcal} / \mathrm{mol} \AA^{3}$, and $z$ is the distance of a gold atom from the wall. The lattice constant (distance of layers) in 


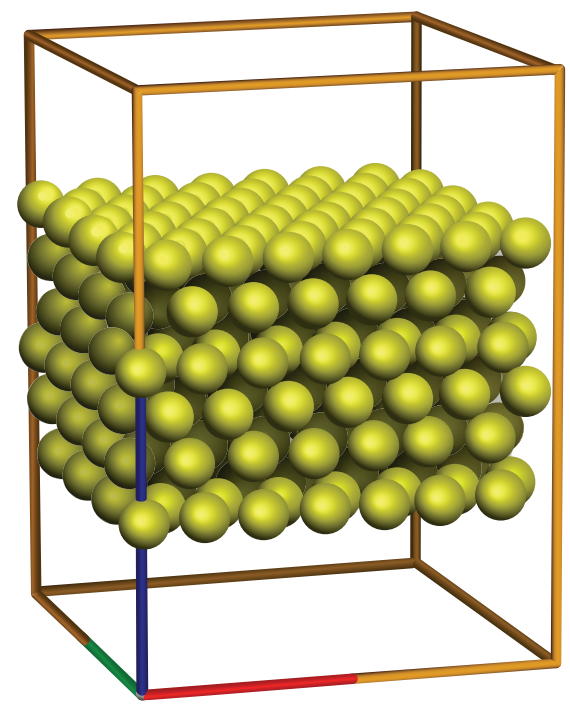

Figure 1: Slab geometry. The system is periodic in the horizontal ( $x$ and $y$ ) directions whereas the $z$-direction is perpendicular to the (111) plane.

the $z$-direction was determined from the $z$-density profile with the exception of two layers from both sides. The peaks of individual gold layers in the slab geometry are clearly discernible because the center of mass of the slab in the $z$ direction is fixed. The positions of the layers are calculated from the centers of mass of the peaks. In some cases of the nanolayer on a substrate, the peaks overlap; the individual peaks are then separated by minima between peaks on a smoothed $z$-density profile. The lattice constant in the $x$ and $y$ directions was determined from averaged $x$ and $y$ sizes of the periodic box. The reference bulk lattice constant was obtained from a standard simulation in a cubic periodic box. Both used potentials exhibit a fast exponential decay of the potential with distance and therefore the simulation box size in the periodic direction may be short. We used a layer of 56 atoms (see Figure 1) in most simulations. For testing purposes we also tried a layer of 90 atoms and did not observe any difference.

2.3. Measurement Techniques. UV-Vis absorption spectra were measured using PerkinElmer's Lambda 25 UV-Vis-NIR spectrometer in the spectral range of 300-900 $\mathrm{nm}$ at scanning rate of $240 \mathrm{~nm} \cdot \mathrm{min}^{-1}$ and a data collection interval of $1 \mathrm{~nm}$. A pristine glass slide was used for background measurement. The typical data uncertainty obtained under this arrangement is below $\pm 5 \%$. Measured spectra were also interpreted in the frame of Tauc's model [26] using Tauc's equation:

$$
\alpha(v)=A \frac{\left(h v-E_{g}^{\mathrm{opt}}\right)^{x}}{h v},
$$

where $\alpha$ is the absorption coefficient of the substance, $E_{g}^{\mathrm{opt}}$ is the substance optical band gap, $x$ is the parameter that gives the type of electron transition, and factor $A$ depends on the transition probability and can be assumed to be constant within the optical frequency range [26]. The optical band gap width, $E_{g}^{\mathrm{opt}}$, of layers was assessed from the linear part of plot $\left((\alpha(v) \cdot h v)^{x}\right.$ versus $\left.h v\right)$. Indirect transitions cannot be excluded in these layers, and therefore $x=2$ were used in the calculation.

$\mathrm{XRD}$ analysis was performed by the automatic powder diffractometer Panalytical X'Pert PRO, equipped with ultrafast semiconductor detector PIXcel, using copper X-ray tube $\left(\lambda_{\mathrm{CuK}} \alpha_{1}=0.1540598 \mathrm{~nm}\right)$. Measurements were performed in a symmetric Bragg-Brentano geometry. Diffraction patterns were registered in the angular range $2 \vartheta=\left(10-85^{\circ}\right)$. Lattice parameter $a$ of cubic face centered lattice of Au was calculated from diffraction lines location and their intensities, using the Rietveld method.

The surface morphology of glass and gold sputtered glass was examined by atomic force microscopy (AFM) using VEECO CP II setup; surface roughness $\left(R_{a}\right)$ was measured in tapping mode with silicon P-doped probes RTESPA-CP, with the spring constant $0.9 \mathrm{Nm}^{-1}$. By the repeated measurements of the same region $\left(1 \times 1 \mu \mathrm{m}^{2}\right.$ in area), we prove that the surface morphology did not change after three consecutive scans.

The chemical structure of gold layers was determined from X-ray photoelectron spectra (ARXPS), measured on Omicron Nanotechnology ESCAProbeP spectrometer. X-ray source was monochromated at $1486.7 \mathrm{eV}$ and area $2 \times 3 \mathrm{~mm}^{2}$ was exposed and analyzed. Spectra were measured stepwise with a step in binding energy $0.05 \mathrm{eV}$; the take-off angle was $0^{\circ}$ and $81^{\circ}$, respectively, according to surface normal. The spectra evaluation was carried out by CasaXPS software. Before the measurement the samples were stored under standard laboratory conditions.

\section{Results and Discussion}

Physicochemical properties of gold nanostructures deposited on glass were studied by several analytical techniques to obtain complex results about studied materials and tested experimental conditions.

UV-Vis spectra of Au/glass sputtered for the sputtering times 20 and $150 \mathrm{~s}$ using currents ranging from 10 to $40 \mathrm{~mA}$ are shown in Figure 2. The thickness (in nm) of the deposited nanostructures which was measured by the scratch test method with AFM [17] is presented in Figure 2. According to expectations, an increase in absorbance of the nanostructures with increasing thickness of the deposited Au layer is observed [27]. The spectra show absorption minimum at ca $500 \mathrm{~nm}$ which exhibits a blue shift with increasing thickness of the Au layer. Dependence of the optical absorption of island-like noncontinuous Au structures on the nanocluster density has been described in [28]. The width of the optical band gap $E_{g}^{\text {opt }}$ was calculated from the UV-Vis spectra using the Tauc equation [26]. The thickness of prepared gold layers varies from $1.0 \mathrm{~nm}(20 \mathrm{~s}$ and $10 \mathrm{~mA})$ to $60.4 \mathrm{~nm}(150 \mathrm{~s}$ and $40 \mathrm{~mA}$ ) and the calculated $E_{g}^{\mathrm{opt}}$ lies between 0.5 and $1.8 \mathrm{eV}$.

The calculated values of the $E_{g}^{\text {opt }}$ are comparable with data published earlier [29]. The calculated values indicate 


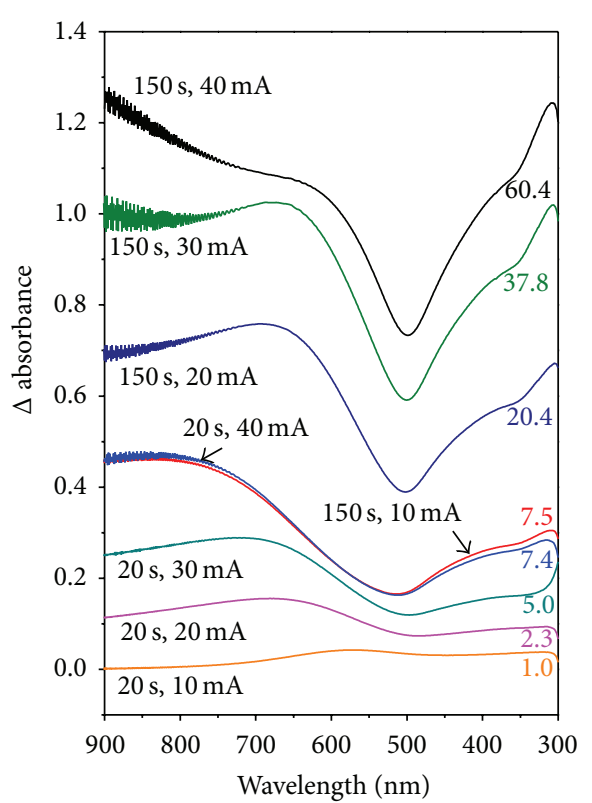

FIGURE 2: UV-Vis spectra of gold films deposited on glass (sputtering times 20 and $150 \mathrm{~s}$ and discharge currents 10,20,30, and $40 \mathrm{~mA}$ ). The numbers on right-hand side are thicknesses of Au layer in $\mathrm{nm}$.

that the $E_{g}^{\text {opt }}$ decreases with increased power and the time of sputtering and this trend corresponds to those in the values of the electrical sheet resistance [17].

XRD-diffraction patterns of the gold nanostructures on the glass by different times and sputtering currents are shown in Figure 3. The intensity of gold diffraction lines is an increasing function of the sputtering time and current. The intensity increase is connected with ever increasing thickness of the gold structures. For currents 10 and $20 \mathrm{~mA}$ and the sputtering time $20 \mathrm{~s}$ the thickness of the gold nanostructures is low and therefore an amorphous line of glass $\left(\mathrm{SiO}_{2}\right)$ with a high width is noticeable in the diffraction pattern. Signal of gold nanostructure in the diffraction pattern becomes observable for sputtering currents above $30 \mathrm{~mA}$. The gold sputtering on glass substrate leads to formation of polycrystals with a preferential orientation in the [111] direction. This is explained by the fact that isolated Au nanoclusters try to minimize their free energy during their growth. This preferential orientation is observed on all samples regardless of sputtering time and current.

Lattice parameter was calculated from diffraction lines parameters using the Rietveld method. It has been published elsewhere [30,31] that the lattice parameter of metals prepared in the form of a thin layer by a physical vapor deposition is not a material constant but depends strongly on the layer thickness. Figure 4 shows the dependence of the lattice parameter and biaxial tension of the gold films on the sputtering time for different sputtering currents derived from the present XRD measurements. The thickness of the deposited gold film is an increasing function of the sputtering time and the discharge current [17]. Monotonous decrease of the lattice parameter with increasing layer thickness is

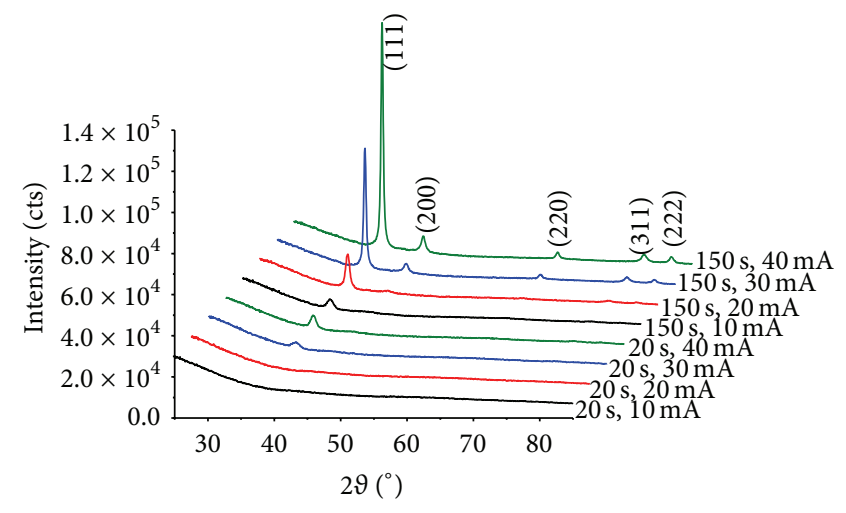

FIGURE 3: 3D XRD patterns of the gold nanostructures coated glass. The samples were sputtered for 20 and $150 \mathrm{~s}$ at the discharge currents $10,20,30$, and $40 \mathrm{~mA}$.

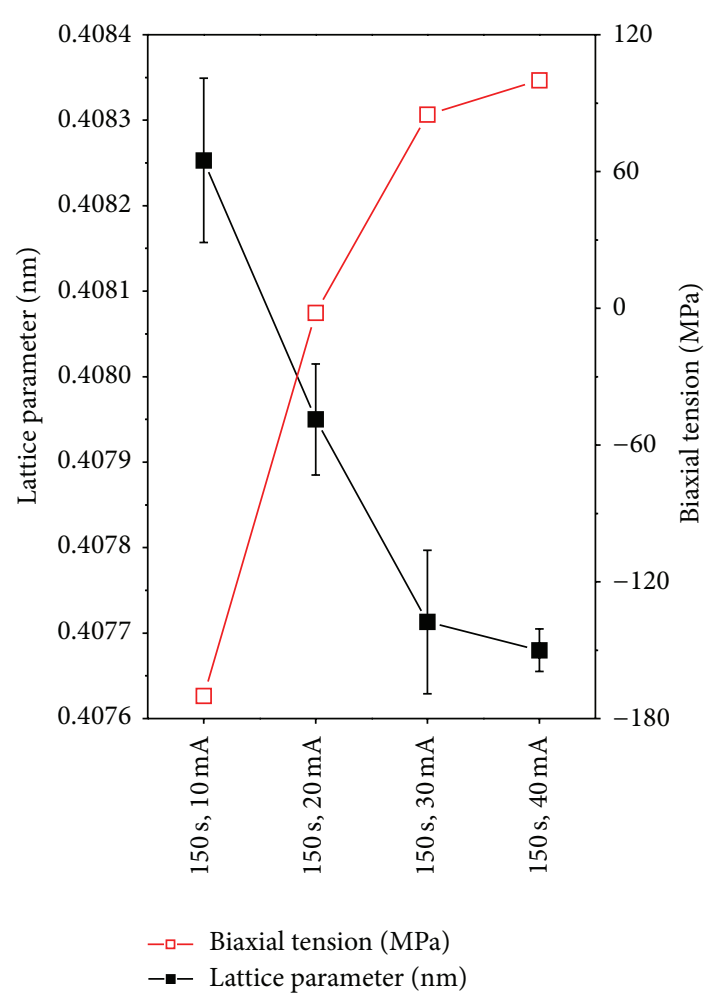

FIGURE 4: Dependence of lattice parameter and biaxial tension on the gold sputtering time and on discharge current.

observed. A possible explanation is provided below. Alternatively, this phenomenon may be caused by an increase of the number of isolated gold nanoclusters $[26,32]$, which aggregate and "push" each other, which in turn increases the biaxial tension. On the other hand, the lattice parameter decreases due to internal stress relaxation during nanocluster growth. This result is in good agreement with those of previous studies [30, 31, 33, 34].

The results of calculations of the lattice parameters are summarized in Figure 5. We can see that the simpler pairwise-additive Erkoc potential predicts that a slab shrinks 


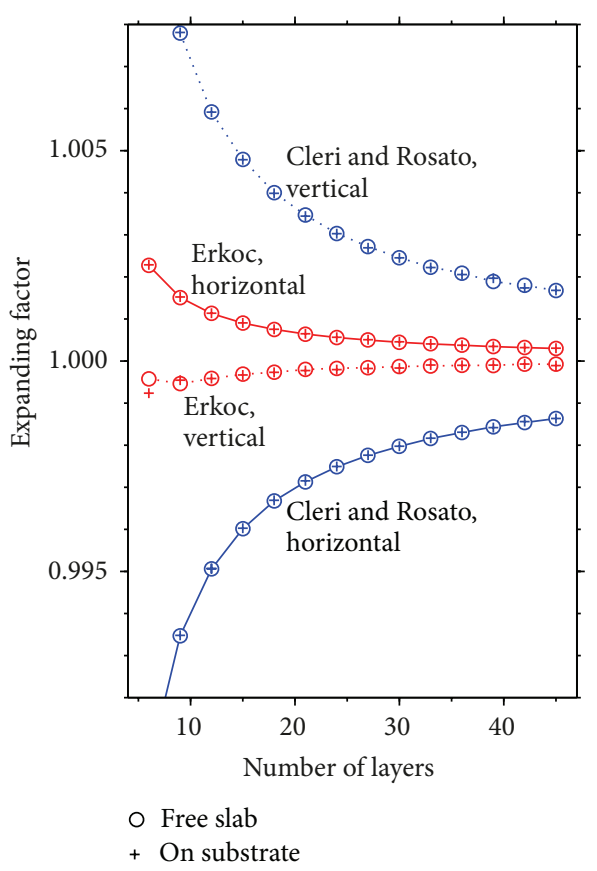

FIGURE 5: Expand factors in directions perpendicular and vertical to the slab with respect to the bulk crystal as the function of the slab thickness.

vertically and expands horizontally. In contrast, the tightbinding potential by Cleri and Rosato predicts horizontal shrinking but expands the interlayer distance in the vertical direction. The latter result is in agreement with our experiment (see above) and with our previous study [33] as well. These results can be qualitatively explained as follows.

First, let us place an atom interacting only by pair forces (Erkoc potential) close to the surface. Because there are more neighbouring atoms in the bulk, the atom is attracted towards the slab centre. The interlayer distance in the vertical direction therefore decreases, in disagreement with experiment. However, there is a tendency to keep volume constant (because of repulsive forces); therefore, the slab has to expand in the horizontal direction. In fact it expands too much and the density of the surface slab becomes lower than that in the bulk.

Electrons in metals can be considered as freely moving in the averaged field of nuclei (more precisely, nuclei with nonvalence electrons). Since the distance of energy levels decreases if more space is available for the electrons, the electron cloud extends slightly out of the metal surface. Because of the nucleus-electron attraction, the nuclei are pulled out of the surface as well, and therefore the slab distance (in the vertical direction) increases, in agreement with experiment. Similarly, the metal slab shrinks in the horizontal direction to (partly) compensate for the vertical expansion; the density of the slab is higher than in the bulk. From the modelling point of view, these phenomena are described by the nonadditive tight-binding types of potentials, as the Sutton-Chen potential used in this work. A detailed analysis of the slab structure is presented in Figure 6

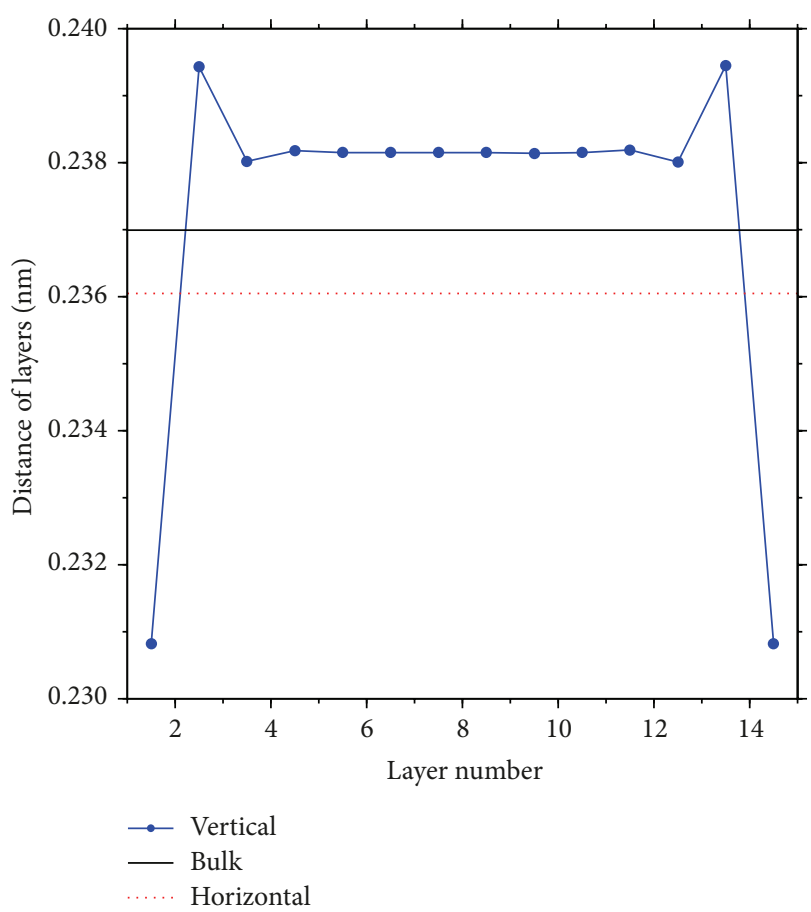

FIGURE 6: Distances between layers in the vertical direction for the slab of 15 layers, modelled by the Cleri-Rosato potential. The black solid line is the bulk value; the dotted red line corresponds to the shrink factor in the horizontal direction.

for a slab of 840 atoms and 15 layers. The blue points correspond to the respective distances between (111) layers. Also shown is the bulk value, $3^{-1 / 2} a$, where $a$ is the cell size. The red dotted line is the "equivalent" horizontal value, namely, $3^{-1 / 2} A^{-1 / 2}$, where $A$ is the area per one atom in a horizontal layer. It can be seen that the outermost layers are most affected and a neat expansion effect in the vertical direction persists inside the slab.

It can be also seen in Figure 5 that the expansion factors are not affected by a substrate within the scale of the graph. Detailed analysis reveals a lower precision of the results, especially in the vertical direction, because the whole nanolayer jumps slightly and randomly above the wall and the peaks of gold layers in the $z$-density profile are asymmetric and sometimes they even overlap. A presence of the substrate slightly decreases the distances between layers for both interatomic potentials; thus, the vertical expansion factor decreases by about $1-2 \times 10^{-5}$ for thinner slabs $(10<$ $N<30$ ), whereas the horizontal expansion factor increases by approximately the same value. To summarize, even if the substrate-metal interactions were seriously underestimated (e.g., because the atomistic structure was neglected), we cannot expect any significant influence of the substrate on the interlayer distances.

Surface morphology and roughness $\left(R_{a}\right)$ of gold nanostructures deposited on the glass substrate were studied by the AFM method and the results are shown in Figure 7. On the 3D AFM scans, a dramatic change in the morphology of the surface with increasing sputtering times and discharge 

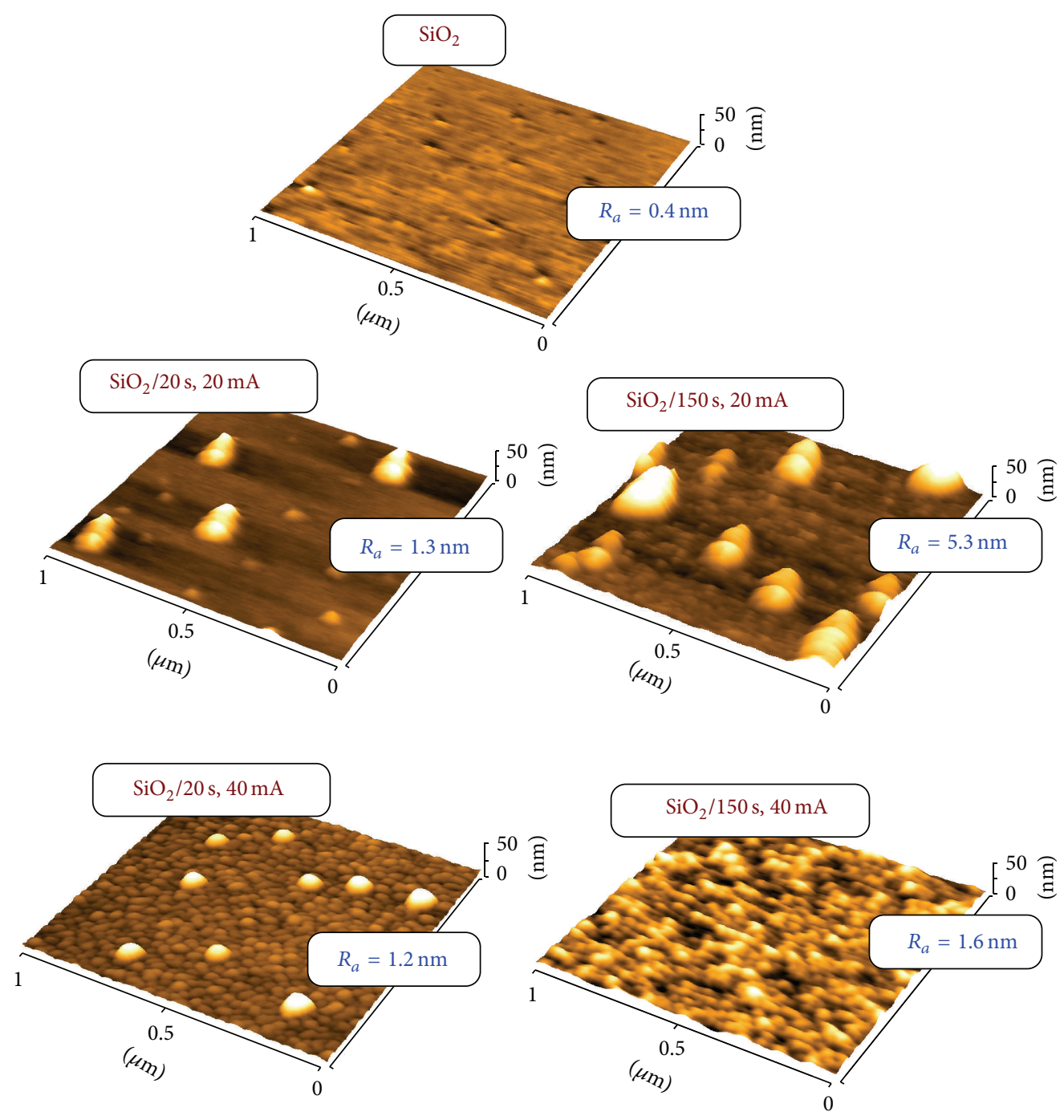

FIgURE 7: AFM images of pristine glass and gold-coated glass, sputtering times 20 and 150 s, and currents 20 and $40 \mathrm{~mA}$.

currents is observed. It is apparent that, in the initial phase of the deposition $(20 \mathrm{~s}$ and $20 \mathrm{~mA}$ ), gold nanoclusters are formed. After $150 \mathrm{~s}$ sputtering time at the same current, electrically continuous gold film is formed [17] with higher surface roughness $\left(R_{a}=5.3 \mathrm{~nm}\right)$. After that, during ongoing deposition, interconnections between nanoclusters are formed and the deposited layers become homogeneous and uniform. Gold nanoclusters are formed on the homogeneous layer with increasing discharge current (20s and $40 \mathrm{~mA}$ ). Surface becomes fully covered by a thin nanolayer of gold after a longer period of deposition (150 s and $40 \mathrm{~mA}$ ) in accordance with the results of [17]. It was published earlier that, under these experimental conditions, an electrically conductive layer was formed on a sample deposited for a sputtering time of $150 \mathrm{~s}$ with the use of a current of $40 \mathrm{~mA}$ [35].

Results of XPS analyses of gold layers sputtered on glass substrate are presented in Table 1. In XPS spectra traces of $\mathrm{Ti}, \mathrm{Zn}, \mathrm{K}$, and $\mathrm{Na}$ from underlying glass are observed. Rather surprising is the presence of carbon (54 at \% in this case) which may completely be attributed to contamination with hydrocarbons and other carbon-rich compounds from ambient atmosphere [36]. As could be expected, the Si signal is a decreasing function of the increasing thickness of gold layer. From XPS measurement under the angle $0^{\circ}$ (analysis depth of 6-8 gold layers) one can see that the gold concentration increases with increasing sputtering time and current. When the sputtering time is increased from 20 to $150 \mathrm{~s}$ the gold concentration increases from 22.5 to $51.9 \%$ and from 34.7 to $42.8 \%$ for 20 and $40 \mathrm{~mA}$ discharge currents, respectively. On the other hand, the XPS measurement under the angle $81^{\circ}$ (1-2 gold layers analysis depth) and the gold concentration on the sample sputtered at $20 \mathrm{~mA}$ current decreases with increasing deposition time. The effect may be due to the presence of gold islands.

From lattice parameter, biaxial tension, and optical bang gap measurements it can be concluded that with increasing time and deposition current $\mathrm{Au}$ nanoclusters are gradually forming electrically and later materially continuous films. The results from simulations support the conclusions of XRD 
TABLE 1: Element concentration from ARXPS measurement accomplished under different angles $\left(0\right.$ and $\left.81^{\circ}\right)$ in gold nanostructures deposited on glass $\left(\mathrm{SiO}_{2}\right)$ after sputtering times 20 and $150 \mathrm{~s}$ and discharge currents 20 and $40 \mathrm{~mA}$.

\begin{tabular}{|c|c|c|c|c|c|c|c|c|}
\hline \multirow{4}{*}{ Sample } & \multicolumn{8}{|c|}{ Element concentration (at. \%) } \\
\hline & \multicolumn{2}{|c|}{$\mathrm{Si}(2 \mathrm{~s})$} & \multicolumn{2}{|c|}{$\mathrm{C}(1 \mathrm{~s})$} & \multicolumn{2}{|c|}{$\mathrm{O}(1 \mathrm{~s})$} & \multicolumn{2}{|c|}{$\mathrm{Au}(4 \mathrm{f})$} \\
\hline & \multicolumn{8}{|c|}{ Angle $\left(^{\circ}\right)$} \\
\hline & 0 & 81 & 0 & 81 & 0 & 81 & 0 & 81 \\
\hline $\mathrm{SiO}_{2}$ & 17.7 & 10.8 & 23.4 & 68.7 & 49.5 & 18.2 & - & - \\
\hline $20 \mathrm{~mA} / 20 \mathrm{~s}$ & 11.9 & 1.8 & 34.1 & 58.3 & 31.5 & 3.6 & 22.5 & 36.3 \\
\hline$/ 150 \mathrm{~s}$ & 3.0 & 0.8 & 39.2 & 64.0 & 5.9 & 7.0 & 51.9 & 17.9 \\
\hline $40 \mathrm{~mA} / 20 \mathrm{~s}$ & - & - & 53.5 & 68.8 & 11.8 & 10.4 & 34.7 & 20.8 \\
\hline$/ 150 \mathrm{~s}$ & - & - & 48.9 & 65.5 & 8.3 & 6.3 & 42.8 & 28.2 \\
\hline
\end{tabular}

analysis. XPS measurements show decreasing concentration of $\mathrm{Si}$ and increasing concentration of $\mathrm{Au}$ which is again connected to formation of continuous Au layer. This process of transition from isolated nanoclusters to continuous films was directly observed using AFM methods.

\section{Conclusions}

Gold nanostructured layers of different thickness and morphology were prepared by sputtering on the surface of glass substrate. The properties of the layers were examined by different techniques. With increasing deposition time and discharge current the UV-Vis absorption increases and the optical band gap $E_{g}^{\text {opt }}$ decreases. According to XRD patterns, the gold sputtering leads to formation of polycrystals with a preferential orientation in the [111] direction, which is supported by the results of simulated Au layer growth. The changes in the lattice parameters of gold nanostructures were observed too in accordance with theoretical estimates done using the tight-binding potential by Cleri and Rosato. Gold sputtering changes the surface morphology dramatically and the transition from island-like structure in initial phase of deposition to continuous gold coverage in the later one is observed by AFM method. By XPS method an increase of surface gold concentration with increasing sputtering time was proved. At the same time the observed Si signal decreases as the gold coverage of underlying glass substrate becomes more complete. The gold/glass structures could find applications in, for example, biosensors, electronics, and tissue engineering. While for tissue engineering $\mathrm{Au}$ nanocluster structure is often desirable, applications regarding electronics demand continuous structures. Therefore, in regard to future application, deposition conditions should be considered carefully.

\section{Conflict of Interests}

The authors declare that there is no conflict of interests regarding the publication of this paper.

\section{Acknowledgments}

This work was supported by the GACR under Project 108/12/G108 and CENTEM Project (Jan Riha,
CZ.1.05/2.1.00/03.0088) cofunded by the ERDF as part of the Ministry of Education, Youth and Sports OP RDI Program.

\section{References}

[1] W. Husinsky, A. Ajami, P. Nekvindova, B. Svecova, J. Pesicka, and M. Janecek, "Z-scan study of nonlinear absorption of gold nano-particles prepared by ion implantation in various types of silicate glasses," Optics Communications, vol. 285, no. 10-11, pp. 2729-2733, 2012.

[2] A. Schaub, P. Slepicka, I. Kasparkova, P. Malinsky, A. Mackova, and V. Svorcik, "Gold nanolayer and nanocluster coatings induced by heat treatment and evaporation technique," Nanoscale Research Letters, vol. 8, pp. 249-256, 2013.

[3] K. L. Kelly, E. Coronado, L. L. Zhao, and G. C. Schatz, "The optical properties of metal nanoparticles: the influence of size, shape, and dielectric environment," Journal of Physical Chemistry B, vol. 107, no. 3, pp. 668-677, 2003.

[4] V. Švorčík, O. Kvítek, J. Ríha, Z. Kolská, and J. Siegel, "Nanostructuring of sputtered gold layers on glass by annealing," Vacuum, vol. 86, no. 6, pp. 729-732, 2012.

[5] S. Guo and E. Wang, "Synthesis and electrochemical applications of gold nanoparticles," Analytica Chimica Acta, vol. 598, no. 2, pp. 181-192, 2007.

[6] M. H. Rashid, R. R. Bhattacharjee, A. Kotal, and T. K. Mandal, "Synthesis of spongy gold nanocrystals with pronounced catalytic activities," Langmuir, vol. 22, no. 17, pp. 7141-7143, 2006.

[7] S. Nakao, T. Ando, M. Shikida, and K. Sato, "Mechanical properties of a micron-sized SCS film in a high-temperature environment," Journal of Micromechanics and Microengineering, vol. 16, no. 4, pp. 715-720, 2006.

[8] E. Hutter and J. H. Fendler, "Exploitation of localized surface plasmon resonance," Advanced Materials, vol. 16, no. 19, pp. 1685-1706, 2004.

[9] E. Bonderover and S. Wagner, "A woven inverter circuit for etextile applications," IEEE Electron Device Letters, vol. 25, no. 5, pp. 295-297, 2004.

[10] J. D. Mendelsohn, S. Y. Yang, J. Hiller, A. I. Hochbaum, and M. F. Rubner, "Rational design of cytophilic and cytophobic polyelectrolyte multilayer thin films," Biomacromolecules, vol. 4, no. 1, pp. 96-106, 2003.

[11] V. Nazabal, E. Fargin, C. Labrugère, and G. Le Flem, "Second harmonic generation optimization in thermally poled borophosphate glasses and characterization by XANES and XPS," Journal of Non-Crystalline Solids, vol. 270, no. 1-3, pp. 223-233, 2000. 
[12] S. Lal, N. K. Grady, J. Kundu, C. S. Levin, J. B. Lassiter, and N. J. Halas, "Tailoring plasmonic substrates for surface enhanced spectroscopies," Chemical Society Reviews, vol. 37, no. 5, pp. 898-911, 2008.

[13] B. D. Todd and R. M. Lynden-Bell, "Surface and bulk properties of metals modelled with Sutton-Chen potentials," Surface Science, vol. 281, no. 1-2, pp. 191-206, 1993.

[14] W. Qi, B. Huang, and M. Wang, "Bond-length and -energy variation of small gold nanoparticles," Journal of Computational and Theoretical Nanoscience, vol. 6, no. 3, pp. 635-639, 2009.

[15] W. J. Huang, R. Sun, J. Tao, L. D. Menard, R. G. Nuzzo, and J. M. Zuo, "Coordination-dependent surface atomic contraction in nanocrystals revealed by coherent diffraction," Nature Materials, vol. 7, no. 4, pp. 308-313, 2008.

[16] W. H. Qi, B. Y. Huang, M. P. Wang, Z. M. Yin, and J. $\mathrm{Li}$, "Molecular dynamic simulation of the size- and shapedependent lattice parameter of small Platinum nanoparticles," Journal of Nanoparticle Research, vol. 11, no. 3, pp. 575-580, 2009.

[17] A. Reznickova, Z. Novotna, N. S. Kasalkova, and V. Svorcik, "Gold nanoparticles deposited on glass: physicochemical characterization and cytocompatibility," Nanoscale Research Letters, vol. 8, no. 1, pp. 252-259, 2013.

[18] V. Švorčík, J. Siegel, P. Šutta et al., "Annealing of gold nanostructures sputtered on glass substrate," Applied Physics A: Materials Science and Processing, vol. 102, no. 3, pp. 605-610, 2011.

[19] F. Cleri and V. Rosato, "Tight-binding potentials for transition metals and alloys," Physical Review B, vol. 48, no. 1, pp. 22-33, 1993.

[20] Ş. Erkoç, "An empirical many-body potential energy function constructed from pair-interactions," Zeitschrift für Physik D: Atoms, Molecules and Clusters, vol. 32, no. 3, pp. 257-260, 1994.

[21] M. P. Allen and D. J. Tildesley, Computer Simulation of Liquids, Clarendon Press, Oxford, UK, 1986.

[22] Ş. Erkoç, "Stability of gold clusters: molecular-dynamics simulations," Physica E: Low-Dimensional Systems and Nanostructures, vol. 8, no. 3, pp. 210-218, 2000.

[23] E. Ricci and R. Novakovic, "Wetting and surface tension measurements on gold alloys," Gold Bulletin, vol. 34, no. 2, pp. 41-49, 2001.

[24] S. Merabia, S. Shenogin, L. Joly, P. Keblinski, and J.-L. Barrat, "Heat transfer from nanoparticles: a corresponding state analysis," Proceedings of the National Academy of Sciences of the United States of America, vol. 106, no. 36, pp. 15113-15118, 2009.

[25] A. K. Rappé, C. J. Casewit, K. S. Colwell, W. A. Goddard III, and W. M. Skiff, "UFF, a full periodic table force field for molecular mechanics and molecular dynamics simulations," Journal of the American Chemical Society, vol. 114, no. 25, pp. 10024-10035, 1992.

[26] J. Tauc, Amorphous and Liquid Semiconductors, Springer, Heidelberg, Germany, 1974.

[27] J. Siegel, O. Kvítek, O. Lyutakov, A. Řezníčková, and V. Švorčík, "Low pressure annealing of gold nanostructures," Vacuum, vol. 98, pp. 100-105, 2013.

[28] R. H. Doremus, "Optical properties of thin metallic films in island form," Journal of Applied Physics, vol. 37, no. 7, pp. 27752781, 1966.

[29] S.-J. Wang, B.-P. Zhang, L.-P. Yan, and W. Deng, "Microstructure and optical absorption properties of Au-dispersed $\mathrm{CoO}$ thin films," Journal of Alloys and Compounds, vol. 509, no. 19, pp. 5731-5735, 2011.
[30] W. Fischer, H. Geiger, P. Rudolf, and P. Wissmann, "Structure investigations on single-crystal gold films," Applied Physics, vol. 13, no. 3, pp. 245-253, 1977.

[31] D. Hazra, S. Datta, M. Mondal, J. Ghatak, P. V. Satyam, and A. K. Gupta, "Thickness dependent lattice expansion in nanogranular Nb thin films," Journal of Applied Physics, vol. 103, no. 10, Article ID $103535,2008$.

[32] J. Siegel, O. Lyutakov, V. Rybka, Z. Kolská, and V. Švorčík, "Properties of gold nanostructures sputtered on glass," Nanoscale Research Letters, vol. 6, pp. 96-104, 2011.

[33] Z. Kolská, J. Říha, V. Hnatowicz, and V. Švorčík, "Lattice parameter and expected density of Au nano-structures sputtered on glass," Materials Letters, vol. 64, no. 10, pp. 1160-1162, 2010.

[34] K. Haeupl, M. Lang, and P. Wissmann, "X-ray-diffraction investigations on ultra-thin gold-films," Surface and Interface Analysis, vol. 9, no. 1-6, pp. 27-30, 1986.

[35] V. Švorčík, P. Slepička, J. Švorčíková, M. Špírková, J. Zehentner, and V. Hnatowicz, "Characterization of evaporated and sputtered thin Au layers on poly(ethylene terephtalate)," Journal of Applied Polymer Science, vol. 99, no. 4, pp. 1698-1704, 2006.

[36] J. Siegel, M. Polivkova, N. Slepickova-Kasalkova, Z. Kolska, and V. Svorcik, "Properties of silver nanostructure-coated PTFE and its biocompatibility," Nanoscale Research Letters, vol. 8, pp. 388397, 2013. 

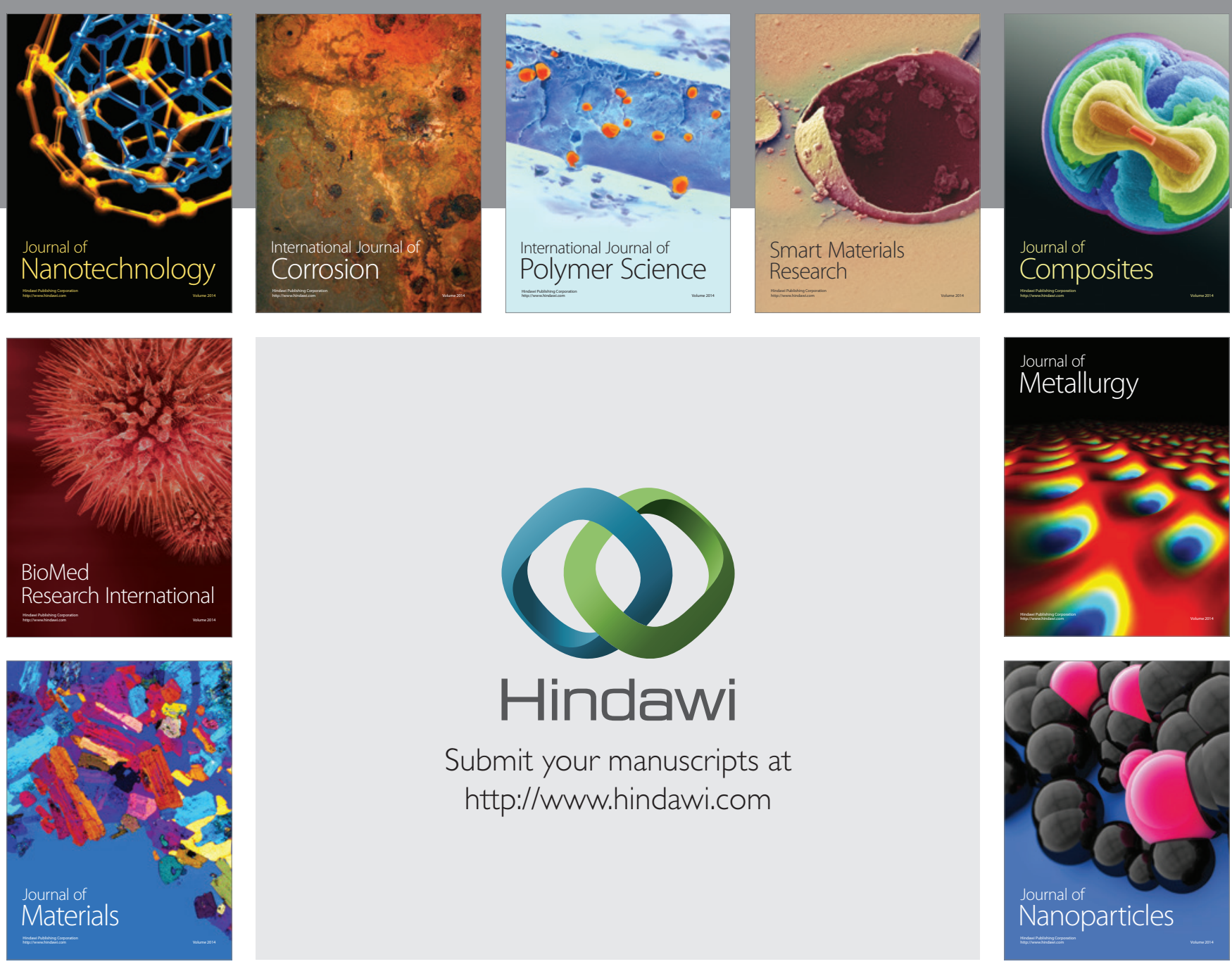

Submit your manuscripts at http://www.hindawi.com
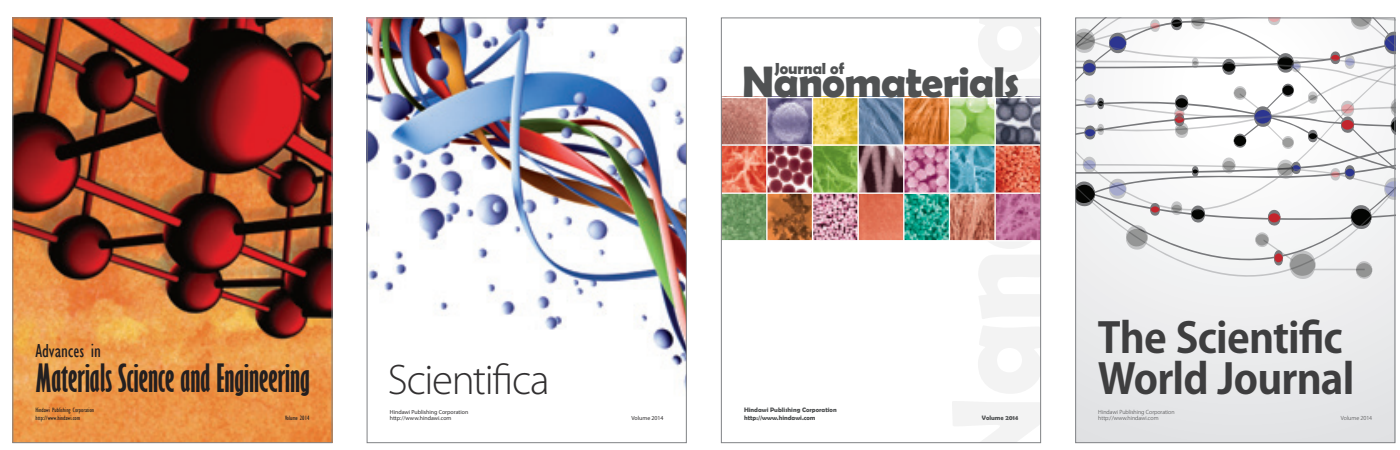

\section{The Scientific World Journal}
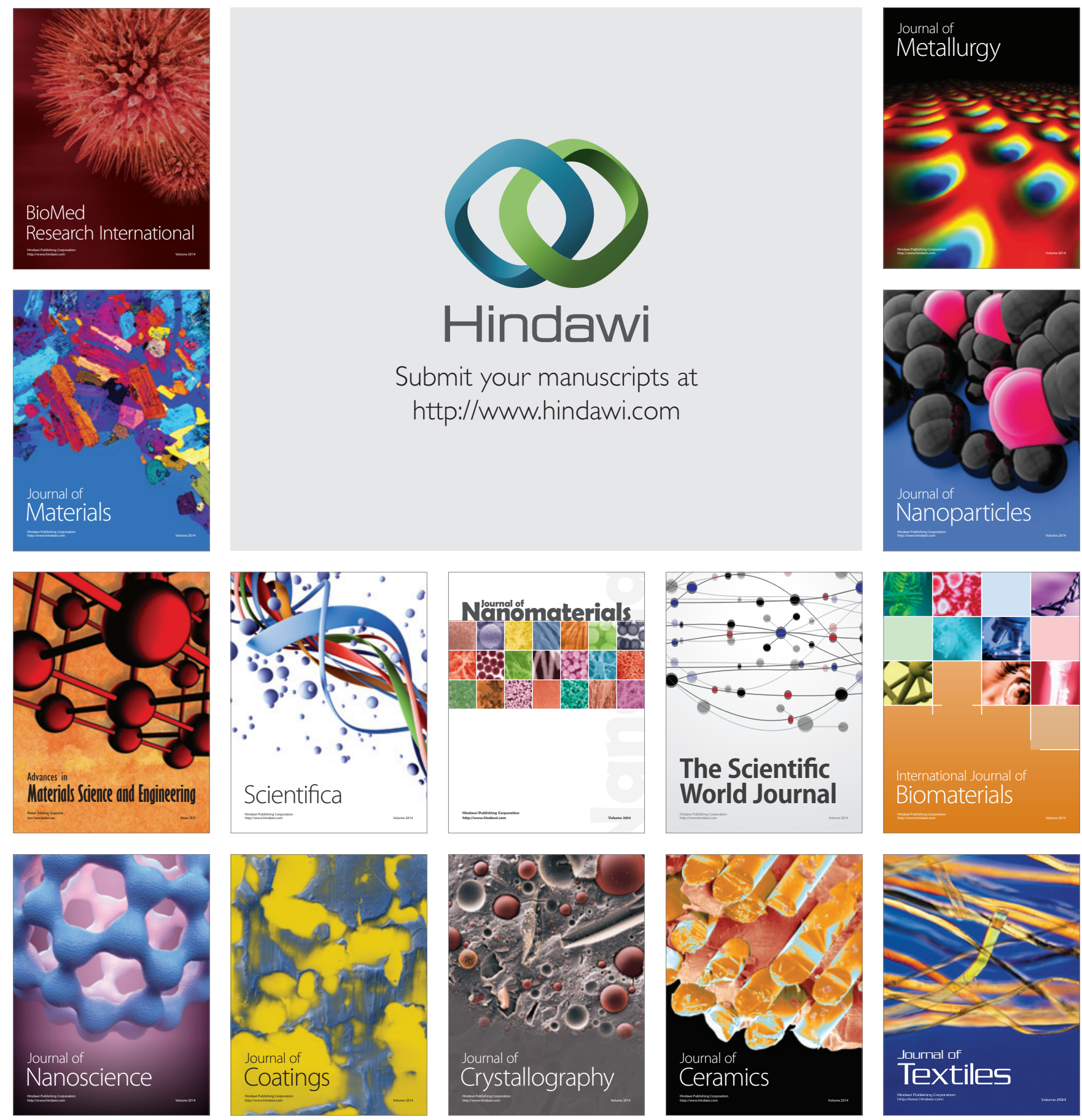